\title{
Congenital Severe Factor V Deficiency: Two Cases with Different Clinical Presentations
}

\author{
Eker $\mathrm{N}^{1 *}$, Tokuç $\mathrm{AG}^{1}$, Yılmaz B ${ }^{2}$, Doğru ${ }^{1}$, Şenay $\mathrm{E}^{2}$, Tufan $\mathrm{B}^{1}$, Aras $\mathrm{S}^{1}$, Koç $\mathrm{A}^{1}$
}

${ }^{1}$ Division of Pediatric Hematology-Oncology, Marmara University, Marmara Medical Faculty, Istanbul, Turkey

${ }^{2}$ Division of Pediatric Hematology-Oncology, Marmara University, Pendik Education and Research Hospital, Istanbul, Turkey

DOI: $10.36347 /$ sjmcr.2020.v08i03.046

| Received: 12.03.2020 | Accepted: 19.03.2020 | Published: 30.03.2020

*Corresponding author: Nurşah EKER

Abstract

Background: Congenital faktor V (FV) deficiency is a rare bleeding disorder in children. The incidence is approximately $1 / 1.000 .000$ in the general population. We aimed to present two patients with congenital FV deficiency who have different clinical presentation and one of them with malignancy. Case report: Case 1 was asymptomatic while Case 2 had intracranial hemorrhage at the time of initial diagnose. Their genetic mutations were different and Case 1 who had a malignancy has heterozygous mutation c.6304 C\&gt;A (p.Arg2102Ser) on 23.exon of F5 which didn't report for FV deficiency before. Conclusion: To the best of our knowledge, there is no report in the literature about the relationship at congenital FV deficiency with malignant diseases. There may be a relationship at this new mutation with malignancy or she might have two diseases coincidentally. In conclusion, we suggest that genotype may play a significant role for severe FV deficiency patients' different clinical presentations.

Keywords: Factor V deficiency, child, malignancy

Copyright @ 2020: This is an open-access article distributed under the terms of the Creative Commons Attribution license which permits unrestricted use, distribution, and reproduction in any medium for non-commercial use (NonCommercial, or CC-BY-NC) provided the original author and source are credited.

\section{INTRODUCTION}

Congenital factor $\mathrm{V}(\mathrm{FV})$ deficiency is a rare bleeding disorder in children. Orwen had first described it in 1947 [1]. The incidence is approximately $1 / 1.000 .000$ in the general population [2]. The corresponding gene for $\mathrm{FV}$ is located on chromosome 1q24, and some mutations in the FV gene leading to FV deficiency can also occur there. This disorder is inherited in an autosomal recessive pattern [3]. Undetectable or low FV levels characterize it, and the clinical presentation is changeable, ranging from asymptomatic to life-threating symptoms such as intracranial hemorrhage. There is prolonged prothrombin time (PT) and activated partial thromboplastin time (aPTT) on laboratory exams due to the decreased FV activity. Fresh frozen plasma (FFP) is used for replacement therapy for FV deficiency patients because recombinant factor replacement therapy is not available yet [4].

We aimed to present two patients with congenital FV deficiency who have a different clinical presentation and one of them with malignancy.

\section{Case 1}

A four-year-old female patient was admitted to our pediatric emergency department with coughing and pain while breathing. She began to cough two weeks ago and then felt pain while breathing. Her symptoms were not accompanied by fever. She and her family did not have a history of bleeding or any severe illness. Physical examination revealed a pulse rate of $92 / \mathrm{min}$, a respiratory rate of $24 / \mathrm{min}$. Lung auscultation revealed tuber souffle on the apex of her right lung. Laboratory examination showed white blood cell count of $4500 / \mathrm{mm}^{3}$, platelet count of $256.000 / \mathrm{mm}^{3}$, and hemoglobin $(\mathrm{Hb})$ of $12,6 \mathrm{~g} / \mathrm{dl}$. There was a well-defined hyperdense area $(3 \times 2 \mathrm{~cm})$ in the upper lobe of the right lung on the PA chest x-ray. Computed tomography (CT) of the thorax showed a soft tissue mass adjacent to the thoracic wall on the right side between 2nd and third ribs (Figure-1). Biopsy of the mass was planned for diagnose. However, the preoperative coagulation studies showed that prothrombin time (PT) and activated partial thromboplastin time (aPTT) were prolonged. The fibrinogen level was within normal limits. The mixing study demonstrated complete correction of the PT and PTT both immediately and after 2 hours of incubation at 37C. This finding was consistent with factor deficiency. The common path 
factor levels (factor II, V, and X) were evaluated. She had decreased FV activity of $0.8 \%$. FII, FVIII, and FX levels were normal. The patient received replacement therapy with FFP, and the biopsy was done. There was no bleeding complication after the biopsy. Final histopathology reported the mass as Ewing sarcoma (ES). There were no distant metastases on her metastatic workup. She received three cycles of neoadjuvant chemotherapy. There were no bleeding complications during the treatment, and she did not receive FFP as prophylaxis or replacement therapy. After three cycles of chemotherapy, CT of the thorax showed nearly complete resolution of the mass lesion. The operation for the total excision of the residual mass was planned. As hemostatic replacement, FFP was transfused before surgery, and then sequential FFP infusions continued every 12 hours for four days. There were no bleeding complications during and after surgery, and the residual mass removed with second and third ribs. On histopathological examination, there was no viable cell, and she completed chemotherapy without complications. She has been followed up with complete remission, and she has had no bleeding symptoms and any replacement therapy for FV deficiency for one year. Her genetic analyzed showed that there were heterozygous mutations c.4203 delC and c.6304 C>A on her FV gene.

\section{CASE 2}

An eight-month-old boy was admitted to our pediatric emergency department with a seizure. He had a history of minor trauma but no history of bleeding. On physical examination, he was pale and lethargic. Laboratory studies showed that Hct: $14.8 \%$, Hgb: 4.9 g/dL, RBC: 2.01x106/ $\mu \mathrm{L}$ MCV: 73.4 fL, WBC: $14.400 / \mu \mathrm{L}$, PT: $43.8 \mathrm{~s}$, INR: 4.88 APTT: $134.4 \mathrm{~s}$, fibrinogen: $355 \mathrm{mg} / \mathrm{dl}$ and electrolytes, hepatic and renal functions were normal. Computed tomography of the brain showed subdural hematoma surrounding the right hemisphere (Figure-2). Mixing studies with normal plasma confirmed factor deficiency. He received erythrocyte suspensions and repeated fresh frozen plasma for replacement therapy after receiving blood to study factor levels. Factor II, FVIII, and FX levels were normal. Plasma FV activity was $0,3 \%$, and he diagnosed with severe FV deficiency. After repeated FFP replacement therapy, the hematoma resolved, and he was discharged without any neurological sequela within ten days. His genetic analysis showed that there were homozygous mutations p.Thr1403Profs*7 (c.4204delC) on his FV gene. The patient has been followed up with FFP prophylaxis for two days a week.

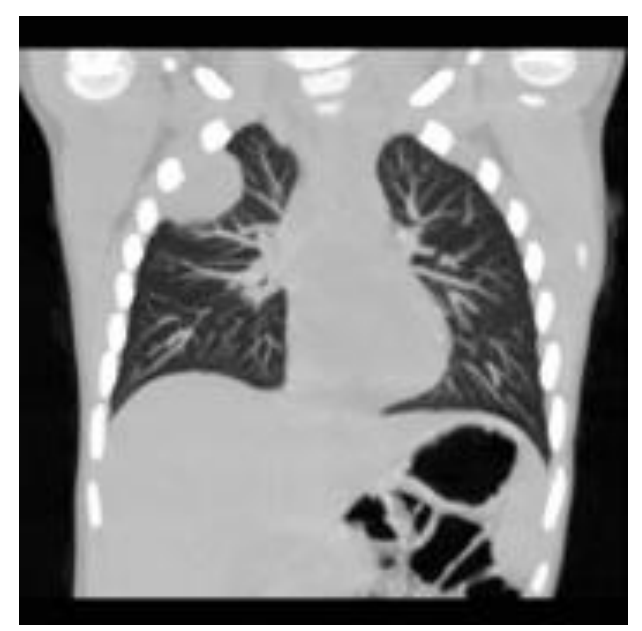

Fig-1: Coronal MIP CT image at Case 1 shows a soft tissue mass adjacent to the thoracic wall on right side between 2 nd and 3rd ribs

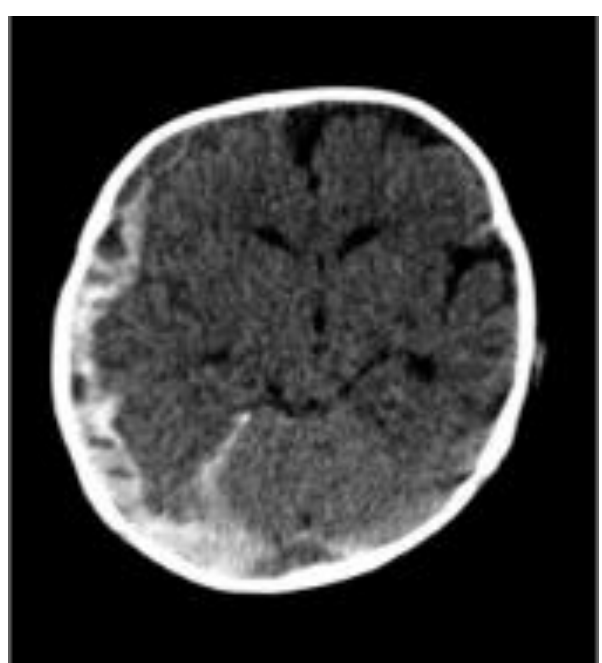

Fig-2: Axial CT image at Case 2 shows subdural hematoma surrounding the right cerebral hemisphere. Multilocation and fluid levels are also noted within hemorrhage

\section{Discussín}

Congenital FV deficiency is a rare bleeding disorder. The clinical presentation of the disease has a range of being asymptomatic to severe bleeding. This clinical manifestation includes epistaxis, hematuria, gum bleeding, haemarthrosis, gastrointestinal bleeding, and postsurgical bleeding [5]. The most common symptoms of FV deficiency are mucosal and soft tissue bleeding, according to studies of Lak et al. and Lucey et al. $[3,6]$. However, severe deficiency may present at birth or early childhood with life-threating bleeding, such as intracranial hemorrhage (ICH). Several studies show that there is no relationship between clinical manifestation and factor level. In the study reported by Safarpour et al. [7] in 2017, 13 patients with FV deficiency were divided into two groups; who has major and minor bleeding. Clinical and laboratory findings analyzed these two groups, and their study identified that there is no relationship between the clinical presentation and plasma factor level. In another study reported by Peyvandi et al. [8] in 2012, the severity of 
bleeding did not correlate with the factor level. Our two patients with severe congenital FV deficiency had different clinical manifestations too. Case 1 was four years old, and she had no history of bleeding, although she had severe FV deficiency. Case 2 was eight months old, and he admitted to our institution with ICH due to severe FV deficiency. The causes of these clinical differences are not clearly understood yet. However, some studies suggest that residual intraplatelets FV has an important role [9]. In a study reported by Duckers et al. in 2010 showed that functional FV in the platelets could protect the severe FV deficiency patients against major bleeding [10]. Our asymptomatic patient (Case 1) may have functional FV in her platelets but we couldn't prove this theory. On the other hand, the bleeding complication was not present even in the period when platelet counts were low because of chemotherapy. Another theory about the differences in clinical findings and factor level is genotype. FV is a large (330 kDa), heavily glycosylated, a single-chain protein similar to FVIII and shares domain organization (A1-A2-B-A3$\mathrm{C} 1-\mathrm{C} 2)$ [11]. The FV gene is approximately $80 \mathrm{~kb}$ in length and contains 25 exons [12]. There are more than 100 DNA mutations on the FV gene, and half of them are missense mutations [13]. FV genes analyzed for both cases. Case 1 has heterozygous mutation c.4203 delC( p.Thr1403Profs*7) on 13.exon of F5, and this mutation identified for FV deficiency before. Also, she has heterozygous mutation c.6304 C>A (p.Arg2102Ser) on 23.exon of F5, too, and this mutation has not been reported for FV deficiency before. This genotype might have a relationship with her clinic presentation. Case 2 has homozygous mutation p.Thr1403Profs*7 (c.4204delC) on 13.exon of F5, this mutation has been identified for FV deficiency before. We suggest that genotype may play a significant role in our patients' different clinical presentations. However, we could not find any report on how these mutations relate to the bleeding situation.

Besides, factor VIII (FVIII) activity must be measured to rule out combined FV and FVIII deficiency. FVIII activity levels were normal in our patients.

Case 1 had a malignancy with FV deficiency. Recent studies show acquired FV deficiency because of autoantibodies is seen in individuals with malignancy. Our patients had decreased FV activity, and the mixing study demonstrated complete correction of the PT and PTT. We excluded FV inhibitors. She had an excellent response to FFP infusion, too. To the best of our knowledge, there is no report in the literature about the relationship of congenital FV deficiency with malignant diseases. Our second patient may be the first case who had congenital FV deficiency and malignant disease. We suggest that she might have had a malignancy with congenital FV deficiency coincidentally.

\section{Conclusion}

Factor V deficiency is a rare bleeding disorder, but it may lead to life-threating symptoms. However, there is no relationship between the plasma factor level and phenotypes of patients for several studies. Clinical and laboratory findings of these two cases support the suggestion that there is no relationship between factor levels and bleeding symptoms, so we suggest each case should be evaluated and followed according to their clinical situation and needs.

\section{REFERENCES}

1. Owren PA. The coagulation of blood: investigation on a new clotting factor. Acta Med Scand. 1947; 194: 11-41.

2. Ellestad SC, Zimmerman SA, Thornburg C, Mitchell TE, Swamy GK, James AH. Severe factor $\mathrm{V}$ deficiency presenting with intracranial haemorrhage during gestation. Haemophilia. 2007; 13: 432-4.

3. Lak M, Sharifian R, Peyvandi F, Mannucci F: Symptoms of inherited factor $\mathrm{V}$ deficiency in 35 Iranian patients. Br J Haematol. 1998;103: 10671069.

4. Naderi M, Eshghi P, Saneei Moghaddam E. Haemophilia. 2013;19:e90-e92.

5. Chen HW, Yeh YT, Tien DM, Yeh CB. Major bleeding as spontaneous haemoperitoneum in a patient with factor $\mathrm{V}$ deficiency. Haemophilia. 2017 Mar;23(2):e158-e160.

6. Mansouritorghabeh H, Manavifar L, Mobalegh A, Shirdel A. Haemorrhagic manifestations and prevalence of factor $\mathrm{V}$ deficiency in Nort-Eastern Iran. Haemophilia. 2010;16: 372-85.

7. Safarpour MM, Haghpanah S, Meshksar A, Karimi M. Phenotype Report on Patients with Congenital Factor V Deficiency in Southern Iran: Recent Ten Years' Experience. Turk J Haematol. 2017 Aug 2;34(3):250-253.

8. Peyvandi F, Menegatti M, Palla R. Rare bleeding disorders: worldwide efforts for classification, diagnosis, and management. InSeminars in thrombosis and hemostasis. 2013 Sep 39; 06: 579584). Thieme Medical Publishers.

9. Duckers C, Simioni P, Rosing J, Castoldi E. Advances in understanding the bleeding diathesis in factor $\mathrm{V}$ deficiency. $\mathrm{Br} J$ Haematol. 2009;146:17-26.

10. Duckers C, Simioni P, Spiezia L. Residual platelet factor $\mathrm{V}$ ensures thrombin generation in patients with severe congenital factor $\mathrm{V}$ deficiency and mild bleeding symptoms. Blood. 2010;115:879-886.

11. Thalji N, Camire RM. Parahemophilia: new insights into factor V deficiency. Semin Thromb Hemost. 2013;39:607-12. 